\title{
Mechanism of Syntaxin Clustering near Secretory Granules in live cells.
}

\author{
Xi Chen ${ }^{1}$, Lei Wan ${ }^{2}$ and Wolfhard Almers ${ }^{3 *}$. \\ 1. Vollum Institute, Oregon Health and Sciences University, Portland, USA \\ 2. Dept. Physiology and Pharmacology, Oregon Health and Sciences University, Portland, USA \\ 3. Vollum Institute, Oregon Health and Sciences University, Portland, USA \\ * Corresponding author: almersw@ohsu.edu
}

Before secretory granules can fuse with the plasma membrane, they must dock there and recruit from it an "acceptor complex" that includes the proteins syntaxin, SNAP-25 and probably Munc18. Interestingly, each of the three proteins can form clusters in membranes, as seen with syntaxin and SNAP25 in immunostained plasma membrane sheets from sonicated PC12 cells [1]. Syntaxin clusters may co-localize with granules if granules are present, but form even without secretory granules [2], even in artificial lipid bilayers, and even when syntaxin's entire cytosolic domain is missing except for 8 "juxtamembrane" residues [3]. Three factors are thought to drive syntaxin cluster formation [4]. (1) In the hydrocarbon core of the lipid bilayer, syntaxin transmembrane domains seek out each other in preference to phospholipid molecules. (2) In the polar layer of the bilayer's internal leaflet, syntaxin's positively charged juxtamembrane residues co-clusters with negatively charged PIP2. (3) In the cytosol, the socalled SNARE domain of syntaxin homo-oligomerizes. None of the three factors are stoichiometric. Is clustering entirely a macroscopic property of syntaxin in a lipid bilayer, or can it occur with molecular specificity, e.g. granules?

Where granules populate the plasma membrane sparsely enough to be distinguished individually, they can be seen to recruit syntaxin, SNAP-25 and Munc18 in live cells [5-8]. In time-resolved recordings, clusters appear where single granules dock at the plasma membrane, and disperse again when granules depart from it [8]. Clearly, granules and clusters interact specifically. We have explored which parts of the syntaxin molecule are needed for its recruitment. Live PC12 cells were imaged by two-color TIRF microscopy. Neuropeptide Y-mCherry marked granules in red, and GFP at the external end of syntaxin (Stx-GFP) marked clusters in green. In images of single cells (Fig. 1A), both the red and green fluorescence appeared punctate, with red dots representing single granules (left) and green dots syntaxin clusters (right). An algorithm located solitary granules in the red image, and excised small square images centered on them. Squares of the same size and at the same locations were excised also in the green image. The resulting image pairs were averaged for all granule sites in the cell (Fig. 1B). Each of the paired images shows a central spot. They represent the averaged granule (left, not analyzed further) and the averaged Stx-cluster (right). We measured the fluorescence of the Stx-GFP spot (1) and that in a narrow annulus (2) surrounding the spot (Fig. 1B, right). (2) represents the local surface density of off-granule Stx-GFP, and the difference between (1) and (2) represents on-granule Stx-GFP [8]. When fluorescence values in this cell were converted into numbers of GFP molecules [9], it appeared that the granule carried an average 18 Stx-GFP molecules and the membrane an average $163 \mathrm{Stx}$-GFP molecules/micron ${ }^{2}$. With 540 molecules/micron ${ }^{2}$ of endogenous syntaxin in our cells [9], Stx-GFP was clearly in competition with an excess of endogenous GFP in our experiment.

Most of a large number of syntaxin mutants diminished the fluorescence of Stx clusters, some to undetectable levels. The cluster brightness reflects a mutant's success in competing with endogenous syntaxin for sites on granules. Together with the surface density of off-granule 
syntaxin, it allows us to estimate the affinity of a mutant to granules. Our main findings are as follows.

(1) In vitro, mutants diminish the binding of Stx to Munc18 with potencies varying over a wide range [10]. These mutants proportionally diminish also the affinity of Stx to granules.

(2) Effects of one set of mutations could be reversed by a set of additional mutations. This is not expected if the two sets are separated by a large distance, as they would be if Stx existed in its "open" conformation. Evidently syntaxin in clusters is "closed".

(3) The relationship between cluster brightness and Stx-GFP expression level suggests that granules recruit Stx at both high and lower affinity. PC12 cells lacking Munc18 show only low affinity recruitment.

(4) We replaced Stx residues in contact with Munc18 with alanines. Three replacements were vastly more effective than all others, and closed syntaxin forces them into tight proximity. They may form the major site for binding Munc18.

We conclude that granules recruit closed syntaxin in a complex with Munc18. The recruitment is specific and of high affinity. It coexists with non-stoichiometric interactions occurring at lower affinity between syntaxin, the lipid bilayers and other objects in the cell.

\section{References}

[1] T Lang, et al. EMBO J. 20 (2001) p. 2202.

[2] T Lang, M Margittai, H Hölzler, R Jahn. J Cell Biol. 158 (2002) p 751.

[3] A Honigmann et al., Nat Struct Mol Biol. 20 (2013) p 679.

[4] F van den Bogaart, T Lang, R Jahn R. Curr Top Membr. 72 (2013) p 1931.

[5] K Aoyagi et al. J Biol Chem. 280 (2005) p 17346.

[6] T Tsuboi M Fukuda, Mol Biol Cell. (2006) 17 p 2101.

[7] S Barg et al., Proc Natl Acad Sci U S A. 107 (2010) p 20804.

[8] NR Gandasi S Barg, Nat Commun. 9 (2014) p 3914.

[9] Knowles et al., Proc Natl Acad Sci U S A. 107 (2010) p 20810-5.

10] P Burkhardt DA Hattendorf WI Weis D Fasshauer EMBO J. 27 (2008) p 923.

[11] The authors acknowledge funding from the National Institute of Health grant MH060600.
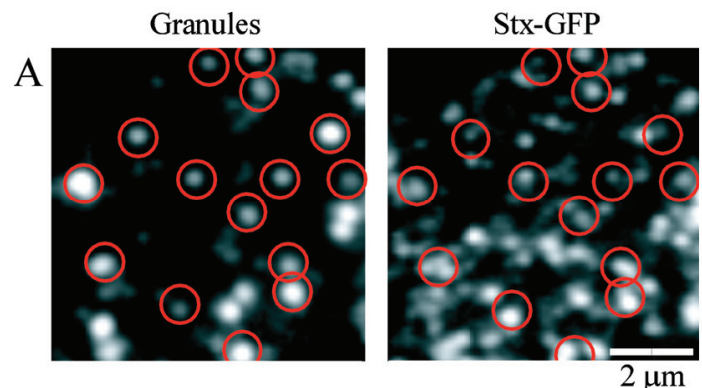

B

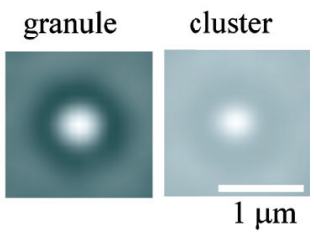

Figure 1. A, portion of a cell showing red (left) and green fluorescence (right). Granule sites were located in the left image and copied into the right (circles). B, average of small square images centered on each of the circles show granule and associated cluster. Because our search algorithm excluded granules with close neighbors, a granule-free zone surrounds each spot as a faint halo. The fluorescence in the right halo represents off-granule syntaxin in the plasma membrane, and reports the surface density of free Stx-GFP. 